\title{
Gamificação como estratégia de aprendizagem ativa no ensino de Física
}

\author{
Gamification as an active learning strategy in the Physics education \\ João Batista da Silva*1일, Gilvandenys Leite Sales², Juscileide Braga de Castro \\ ${ }^{1}$ Instituto Federal de Educação, Ciência e Tecnologia do Ceará, Programa de Pós-Graduação em Ensino de Ciências e \\ Matemática, Fortaleza, CE, Brasil \\ ${ }^{2}$ Instituto Federal do Ceará, Fortaleza, CE, Brasil \\ ${ }^{3}$ Universidade Federal do Ceará, Faculdade de Educação, Departamento Teoria e Prática do Ensino, Fortaleza, CE, Brasil
}

Recebido em 05 de Novembro, 2018. Revisado em 12 de Março, 2019. Aceito em 21 de Março, 2019.

\begin{abstract}
Neste trabalho são apresentados os resultados do ganho de aprendizagem proporcionado pela gamificação aplicada como estratégia de aprendizagem ativa nas aulas de Física com alunos do Ensino Médio. A gamificação consiste na utilização dos elementos de design de jogos no ambiente de aprendizagem para engajar, motivar e melhorar o desempenho dos alunos. Nessa investigação foi realizado um estudo quase-experimental envolvendo grupo controle (GC) e grupo experimental (GE). Os instrumentos utilizados para coleta de dados foram dois testes iguais aplicados antes e depois da aplicação da metodologia. A análise do desempenho de cada grupo foi realizada por meio do teste de ganho normatizado proposto por Hake. Os resultados mostraram que os alunos que tiveram aulas gamificadas obtiveram um ganho de aprendizagem $(\mathrm{g}=0,38)$ superior aos alunos que tiveram aulas tradicionais $(\mathrm{g}=0,11)$. Diante da carência de pesquisas empíricas na literatura que mensurem ganho normatizado em atividades gamificadas no ensino de Física, espera-se que esta pesquisa possa contribuir para cobrir essa lacuna. Palavras-chave: Gamificação, Ensino de Física, Aprendizagem ativa, Ganho de Hake.
\end{abstract}

This work presents the results of the learning gain provided by gamification applied as an active learning strategy in Physics classes with high school students. Gamification consists of using game design elements in the learning environment to engage, motivate, and improve student performance. In this investigation, a quasi-experimental study was carried out involving control group (CG) and experimental group (GE). The instruments used for data collection were two equal tests applied before and after the application of the methodology. The performance analysis of each group was performed through the normalized gain test proposed by Hake. The results showed that the students who had gamified classes achieved a learning gain $(\mathrm{g}=0.38)$ higher than the students who had traditional classes $(g=0,11)$. Given the lack of empirical studies in the literature that measure normalized gain in gamified activities in Physics education, it is hoped that this research may contribute to cover this gap.

Keywords: Gamification, Physics education; Active learning, Normalized gain.

\section{Introdução}

O ensino de Física na educação contemporânea urge por mudanças e grandes são os desafios na Educação Básica: despreparo de professores, más condições de trabalho, número reduzido de aulas, a aprendizagem mecânica de conteúdos desatualizados, ensino centrado no docente, não no aluno, entre outros [1].

Além dos muitos desafios, pesquisas na área de Ensino de Física apontaram que a falta de motivação para aprendizagem tornou-se um dos principais desafios enfrentados pelos professores em sala de aula [2], o que acaba contribuindo com a inviabilização do ensino e, consequentemente, com a aprendizagem da Física.

Uma das razões que tem contribuído para a desmotivação dos alunos é o modelo passivo/expositivo de

*Endereço de correspondência: joaobathista82@hotmail.com aprendizagem, centrado no professor, e que costuma utilizar métodos de resolução de exercícios e estratégias para decorar fórmulas matemáticas, o que apenas servem para as provas de vestibulares $[3,4]$. Contudo, vale destacar que este modelo, atualmente, ainda é um dos paradigmas educacionais mais influentes nas salas de aula do Brasil.

De acordo com Schroeder [3], no modelo de ensino tradicional, os alunos costumam permanecer estáticos, concentrados fazendo atividades/tarefas por longo período de tempo, repetindo sempre o ciclo aulas-exercícios-testes, o que nem sempre é prazeroso. Não obstante, o autor destaca que o aprendizado, além das habilidades cognitivas, depende também de motivação pessoal do aluno para desenvolver outras habilidades como a capacidade de perseverar, de lidar com frustrações (auto-controle) e refletir sobre suas ações e expectativas. Desta forma, faz-se necessário refletir, tanto sobre fenômenos oriundos 
das interações professor-aluno-conhecimento científico, como quanto aos tratamentos que deverão ser aplicados aos conteúdos específicos de Física que se pretendem ensinar $[5,6,7,8]$.

Tratar o conhecimento científico, de maneira a torná-lo potencialmente compreensível pelos alunos, não é uma tarefa tão trival. Principalmente, porque demanda conhecimentos, por parte do professor, sobre alguns elementos (de natureza epistemológica, cognitiva e metodológica) pertinentes ao campo da Didática da Física (DF) $[7,8]$. Tais conhecimentos são essenciais para o processo de transposição didática dos conhecimentos científicos, o qual demanda tempo e dedicação, que nem sempre o professor dispõe. Desta forma, a falta de tempo e/ou dedicação acaba fortalecendo a predominância do ensino tradicional (aulas-exercícios-testes) no Brasil.

Sobre a eficácia desse método de ensino, Silva [9] destacou que este modelo de ensino teve e continua a ter sua importância para o processo educativo, todavia, a sociedade passou por mudanças comportamentais radicais devido ao avanço das Tecnologias Digitais de Informação e Comunicação (TDIC). Considerando que os alunos contemporâneos já não são mais os mesmos para os quais o sistema educacional foi criado, as pesquisas apontam que o método tradicional de ensino, por si só, já não é mais capaz de atender as demandas do aluno contemporâneo [9].

Este cenário tem instigado pesquisadores da área de ensino Física a concentrar esforços no desenvolvimento de novas metodologias de ensino que visam o engajamento dos estudantes [10]. Dentre elas, o uso de metodologias ativas no ensino de Física tem se mostrado uma maneira alternativa para buscar o interesse e, principalmente, a motivação dos alunos no século XXI $[11,12]$.

Müller et al. [10] destacaram que a falta de motivação dos estudantes para aprender os conteúdos escolares abordados, não é um problema apenas do ensino de Física, mas da Educação Básica em geral. Diante da falta de motivação discente, nos últimos anos no Brasil, têm surgido várias pesquisas empíricas sobre a aplicação de metodologias ativas no ensino de Física, entre elas destacam-se: aprendizagem baseada em equipes; aprendizagem baseada em problemas; Peer Instruction [13]; Predict - Observe - Explain (POE) [14, 15]; Investigative Science Learning Environment (ISLE) [16]; e gamificação [17, 18,19, 20].

Dentre estas metodologias, a gamificação vem ganhando destaque nacional e internacional devido a sua capacidade de envolver, engajar e motivar a ação do estudante em ambientes de aprendizagem [14, 19]. Além disso, essa metodologia tem se mostrado como uma alternativa promissora para o ensino de Física [12, 17, 18, 20, 21, 22].

Diferentemente da metodologia Peer Instruction, que foi planejada especificamente para o ensino de Física, a gamificação ainda é uma área incipiente que tem sido adaptada para o ensino de Física [18]. Além disso, vale ressaltar que apesar da maioria das pesquisas apontarem o potencial da gamificação para motivar os alunos nas aulas de Física [12, 17, 18, 20, 21, 22], ainda há uma enorme carência de trabalhos na literatura nacional e internacional que investiguem o ganho normatizado de aprendizagem proporcionado por essa metodologia de ensino. Nesse sentido, o objetivo geral dessa pesquisa é investigar a eficiência da gamificação na aprendizagem nas aulas de Física por meio do teste de ganho normatizado de Hake. Para tanto, na próximas seção, apresentar-se-á uma discussão sobre gamificação. Em seguida, na seção 3 , serão descritos os aspectos metodológicos. Na seção 4 serão apresentados e discutidos os resultados. E por fim, na seção 5, são feitas as considerações finais.

\section{Gamificação}

Nos últimos anos, a quantidade de pesquisas sobre gamificação em ambientes de aprendizagem tem merecido destaque, o que consolida a gamificação como um fenômeno emergente $[12,14,17,18,20,21,22]$.

Por definição, a gamificação contempla o uso de elementos de design de games em contextos fora dos games para motivar, aumentar a atividade e reter a atenção do usuário [23]. Os elementos de games são objetivos, regras claras, feedback imediato, recompensas, motivação intrínseca, inclusão do erro no processo, diversão, narrativa, níveis, abstração da realidade, competição, conflito, cooperação, voluntariedade, entre outros [24].

Aplicar a gamificação é como utilizar várias ferramentas (elementos de games) que estão dentro de uma caixa $[24,25]$, e que podem ser combinadas de diferentes maneiras. Todavia, para sua utilização correta, deve-se conhecer quais são as funções de cada uma e como elas irão interagir dentro do sistema proposto [24]. Contudo, é importante destacar que para gamificar uma atividade não é necessário utilizar todos os elementos de games, mas apenas alguns. Ou seja, pode-se utilizar desde um número reduzido, até uma quantia maior dos elementos [24]. Não obstante, McGonigal [26] destacou que quatro elementos são fundamentais em qualquer jogo: voluntariedade, regras, objetivos e feedbacks.

Conforme ilustrado na Figura 1, no processo de gamificação os elementos devem estar interconectados, fazendo com que o produto final possa produzir uma experiência próxima a de um game completo [24]. A voluntariedade implica na aceitação das regras, objetivos e feedbacks.

O objetivo é o elemento que direciona o participante de um jogo a se concentrar para atingir o propósito. Os objetivos devem ser claros, caso contrário podem tornar o jogo confuso e difícil, conduzindo-o ao fracasso [24]. Além disso, se o objetivo for muito complexo ele deverá ser subdividido em outros menores para tornar o jogador apto a atingir o objetivo maior.

As regras compõem um conjunto de disposições que limitam as ações dos jogadores e condicionam a realização do jogo. Sua função é definir a maneira que o jogador deverá se comportar ou organizar suas ações para o 


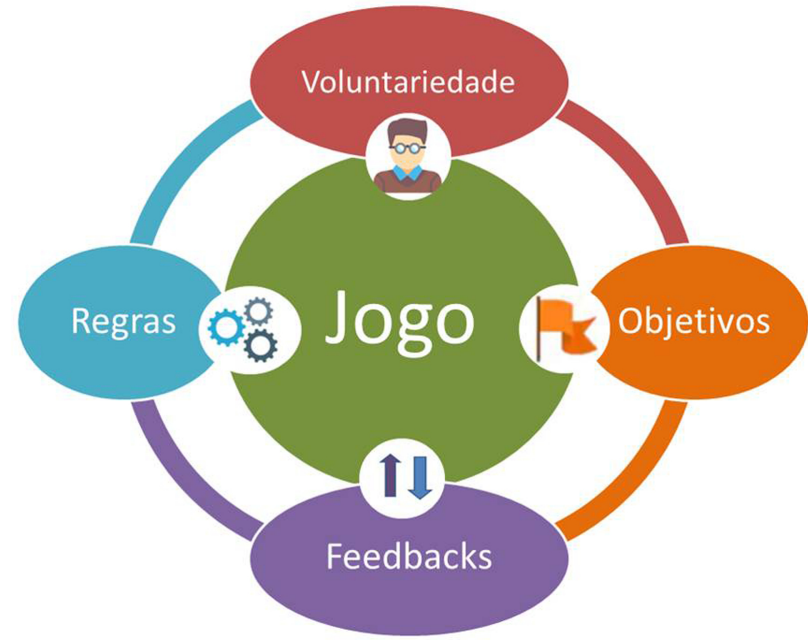

Figura 1: Representação esquemática dos elementos de games interconectados.

cumprimento dos desafios impostos pelo jogo [26]. As regras possibilitam que o jogador explore os espaços oferecidos, para desenvolver a criatividade e motivar o pensamento estratégico.

A função principal dos feedbacks é informar aos jogadores como está sua relação com os diferentes aspectos que regulam sua interação com a atividade para fomentar motivação. Para isso, os feedbacks devem ser imediatos, claros e diretos. Essa é a marca fundamental dos bons games, pois são eles que fornecem continuamente uma visualização aos jogadores de seu estado perante o objetivo do jogo [24]. Em síntese, é a aceitação dos objetivos, regras e feedbacks que viabilizam condições comuns para harmonização em jogos.

É importante destacar que, embora a gamificação utilize os mesmos elementos, esta diverge dos games de entretenimento porque não contempla a jogabilidade [27]. Ademais, outra característica importante sobre a gamificação é que, embora a maioria das pesquisas atuais sobre gamificação aplicada à educação inclua o uso de TDIC em suas práticas, o uso de tecnologias digitais não é uma condição necessária para implementar uma estratégia baseada na gamificação da sala de aula [18, 23, 24, 28].

A estratégia de utilizar elementos de jogos, também tem sido investigada na educação [24] e em particular no ensino de Física [27,29], cujo objetivo é potencializar o processo de aprendizagem Nesse sentido, "a gamificação no contexto educacional consiste na utilização de elementos de design de jogos no ambiente de aprendizagem, não para jogar, mas para motivar, engajar e melhorar o rendimento e desempenho dos alunos envolvidos no processo de ensino" $[18,19]$.

Vale ressaltar que ainda são grandes os desafios para se implementar a gamificação de maneira eficaz em sala de aula. Ademais, apesar da gamificação ter se mostrado uma alternativa promissora para promover motivação intrínseca, engajamento e sentimento de realização [27], um dos desafios na criação de ambientes gamificados é saber como estimular e relacionar efetivamente as duas formas de motivação (extrínseca e intrínseca). Uma aplicação efetiva da gamificação para alcançar resultados satisfatórios demanda um bom planejamento [30], e para isso é necessário um aprofundamento teórico [25, 31].

Dessa forma, para que o professor possa obter sucesso em suas atividades é interessante que ele conheça pelos menos alguns aspectos teóricos subjacentes à gamificação: a teoria da autodeterminação e a teoria do fluxo.

A teoria da autodeterminação (Self-Determination Theory, $S D T$ ) se propõem a explicar os componentes das motivações (extrínseca e intrínseca) e os fatores relacionados com a sua promoção, o que envolve questões epistemológicas relativas a saúde e o bem-estar psicológico [32]. A motivação intrínseca é caracterizada pelo interesse interno do sujeito em si mesmo [3, 32], em se satisfazer ao realizar uma atividade e se envolver voluntariamente para gerar autonomia necessária no aluno. Motivar intrinsecamente os alunos em sala de aula é uma tarefa complexa e desafiadora para o professor, o que demanda tempo e conhecimento teórico. Em suma, a $S D T$ propõe que há três necessidades básicas, inatas, subjacente à motivação intrínseca: autonomia, competência e pertencimento. De acordo com Studart [27] essas necessidades são atendidas nos bons games e, portanto, devem ser incorporadas no processo de gamificação.

A Teoria do Flow, criada pelo psicólogo húngaro Mihaly Csikszentmihalyi em 1991, se propôs a explicar quais são os motivos que levam as pessoas a ficarem completamente envolvidas e concentradas em determinadas atividades que não proporcionam nenhum tipo de retorno material ou financeiro. Em síntese, esta teoria procura descrever o estado mental de operação automatizada, em que o sujeito está completamente imerso em uma sensação de foco energizante (profunda concentração, envolvimento e prazer) ao se envolver em uma atividade específica, na qual há equilíbrio entre o nível de dificuldade do desafio e a habilidade compatível do sujeito, de maneira que permita sua realização com êxito.

Conforme o diagrama ilustrado na Figura 2, há duas dimensões importantes (desafios e as habilidades) durante a experiência de Fluxo, ambas são representadas por eixos do diagrama. Desta forma, ao iniciar as aulas o professor deverá propor aos alunos um desafio básico que seja possível de ser realizado por um aluno que possui habilidade compatível (A1). Sendo assim, logo após a conclusão da atividade, o professor deverá proporcionar um novo desafio que exija um maior nível de habilidade do aluno. O novo desafio não poderá ser tão difícil, que leve o aluno ao estado de ansiedade (A3), e nem tão fácil que possa levá-lo ao tédio (A2).

O equilíbrio entre desafio e habilidade é uma das principais características do Fluxo [34]. Para se alcançar o equilíbrio, toda atividade proposta, que apresenta desafios a serem cumpridos, deve pressupor que o sujeito tenha consciência de que tal atividade é possível de ser 


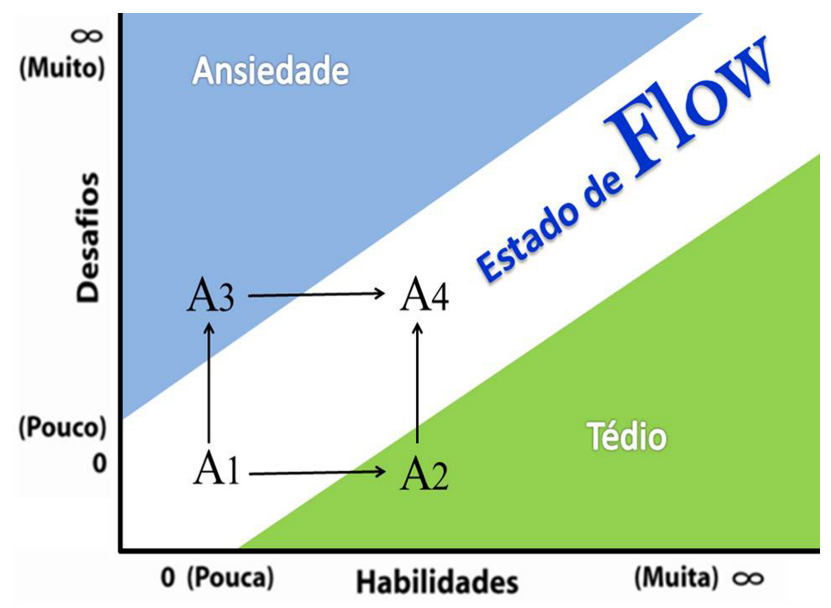

Figura 2: Diagrama esquemático desenvolvido por Mihaly para tentar explicar o estado de Fluxo [29].

feita. Caso contrário, não é vista como um desafio, pois não apresenta sentido em ser realizada [33]. Conforme Csikszentmihalyi [34] cada pessoa possui um nível médio de desafios e habilidades. Assim, o estado de Flow passará a existir quando o aluno enfrentar desafios (situaçõesproblema) diretamente proporcionais às suas habilidades.

Dessa forma, possibilitar que o aluno compreenda qual é sua missão, investigue, faça suas atividades e obtenha sucesso pelo seu próprio esforço é uma das características das metodologias de aprendizagem ativas.

\subsection{Gamificação como estratégia de aprendizagem ativa}

A gamificação não é necessariamente uma metodologia ativa de ensino, todavia, poderá ser utilizada como estratégia de aprendizagem ativa [12]. Uma metodologia de aprendizagem ativa está fundamentada no pressuposto de que o aluno, e não o professor, encontra-se no centro do processo de aprendizagem $[12,35]$. De acordo com Lovato [11], essa metodologia parte da premissa de que o aluno é instigado a sair uma posição cômoda, puramente receptora de informações, para participar ativamente das aulas em um contexto em que poderá desenvolver novas habilidades/competências necessárias como: criatividade, autonomia, iniciativa, a criticidade reflexiva, capacidade de inovar, cooperação para se trabalhar em equipe e refletir diante de situações problemáticas.

No modelo de aprendizagem ativa o professor passará a atuar como mediador, orientador, supervisor e facilitador do processo de aprendizagem [11]. A gamificação como estratégia de ensino pode ser considerada como um exemplo de ensino metodologia ativa quando o professor, por meio de regras claras (contrato didático), desafia e estimula os alunos a realizarem suas missões (tarefas) [12]. Só que para cumprir cada missão os alunos deverão desenvolver algumas estratégias como: a) explorar o problema (desafio); b) levantar hipóteses; c) tentar solucionar o problema a partir de seus conhecimentos prévios; d) identificar o que ele não sabe e o que é preciso conhecer para solucionar o problema; e) determinar as tarefas individuais e delegar responsabilidades para o estudo autônomo da equipe; f) compartilhar o novo conhecimento; f) aplicar o conhecimento para solucionar o problema; e g) avaliar a solução do problema e a eficácia do processo utilizado.

Essa prática de ensino, que possibilitam ao aluno fazer alguma atividade e, ao mesmo tempo, o leva a pensar sobre o que está fazendo, sintetiza um os princípios das metodologias ativas de aprendizagem que é favorecer no aluno as atividades de ouvir, ver, perguntar, discutir, fazer e ensinar [36].

Silva e Sales [18] pontuam que facilitar o desenvolvimento dessas habilidades/competências é uma tarefa árdua e complexa, principalmente na sociedade contemporânea em que o professor deixou de ser a única fonte de conhecimento/informação para os alunos. Desta forma, apenas o método convencional/tradicional de ensino, como aulas meramente expositivas, por si só, já não é mais capaz de atender as demandas educacionais contemporâneas [9]. Além disso, ainda tem o problema da falta de motivação na sala de aula, o qual foi apontado com um dos principais desafios para o ensino de Física.

Considerando que o processo educativo do aluno é fruto da constante interação entre os diversos campos (família, sociedade, momento histórico, filosofia e as tecnologias) em que o sujeito está inserido [18], percebe-se que a escola tem o papel de saber quais são as habilidades necessárias para o aluno contemporâneo que não eram exigidas há décadas atrás.

\section{Metodologia}

A presente pesquisa foi aprovada pelo Comitê de Ética e Pesquisa do Instituto Federal de Educação, Ciência e Tecnologia do Ceará (IFCE), protocolado sob o número CAAE: 74965717.6.0000.5589. A investigação foi realizada com 49 alunos de duas turmas do $2^{\circ}$ ano do Ensino Médio dos cursos de Técnico Integrado do IFCE. Como as turmas já estavam formadas antes da intervenção do pesquisador, não sendo, portanto, possível utilizar a aleatoriedade na escolha dos sujeitos da pesquisa, utilizou-se um delineamento quase-experimental envolvendo grupo experimental (GE) e grupo de controle (GC). Desta forma, o controle dos pesquisadores ficou restrito, unicamente, na decisão sobre qual dos grupos deveria receber o tratamento [37].

Neste delineamento, inicialmente foi aplicado um préteste igual em ambos os grupos. Após a aplicação do teste, o GC, composto por 33 alunos do curso Técnico integrado em Informática, teve aulas tradicionais, ministradas pelo professor A. E o GE, composto por 16 alunos do curso Técnico integrado em eletrotécnica, teve aulas gamificadas, ministradas pelo professor B. 
O GC teve aulas tradicionais, puramente expositivas. Este modelo de ensino priorizou o mesmo método de comunicação, no qual o professor ocupou a posição de protagonista e transmissor da informação. Os recursos didáticos utilizados nas aulas foram: laboratório de Física, lista de exercícios, quadro, pincel e o livro texto.

O GE teve aulas gamificadas nas quais a sala de aula foi organizada em 'ilhas de aprendizagem', cujo objetivo foi proporcionar mais interação entre os alunos. Neste design, o grupo maior foi dividido em subgrupos para possibilitar a exploração de avanços educacionais potencializados pelo uso de TDIC, para proporcionar que os alunos pudessem resolver problemas, individualmente ou em grupo, para realizar atividades práticas e para discutir o material de maneira a facilitar a compreensão do conteúdo por aqueles que não entenderam.

Para sistematizar o processo de ensino, gamificou-se e implementou-se, em fases, uma sequência didática, cujo objetivo foi facilitar a aprendizagem de tópicos específicos de óptica geométrica no Ensino Médio.

A implementação da sequência didática foi dividida em sete passos (Figura 3): definição dos tópicos específicos e o contrato didático; verificação dos conhecimentos prévios dos alunos; aplicação de situações-problema iniciais de nível básico; aplicação de situações-problema de nível intermediário; aplicação de situações-problema de nível avançado; avaliação da aprendizagem; e realização de encontro final integrador.

$1^{\circ}$ passo: Definir tópicos específicos e o contrato didático

Inicialmente apresentou-se aos alunos o plano de ensino da disciplina e os objetivos a serem alcançados, bem como as regras. Explicou-se que o conteúdo seria dividido em fases, cada uma correspondendo a um tópico específico de óptica geométrica (princípios de óptica geométrica, reflexão da luz, refração da luz, fenômenos ópticos, e formação de imagens em espelhos planos) e que ao final de cada tópico seriam realizados quizzes para avaliação da aprendizagem. Ficou esclarecido que as missões (atividades específicas individuais e em times) teriam peso proporcional ao nível de complexidade

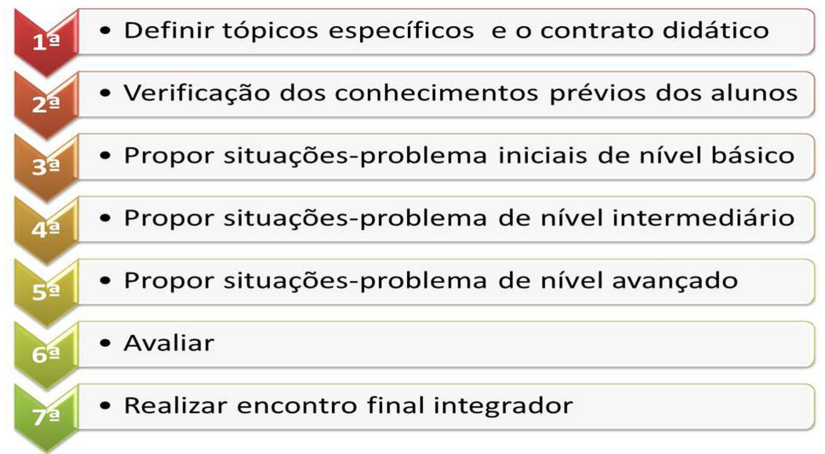

Figura 3: Diagrama esquemático do desenvolvimento e implementação da sequência didática. para sua realização. O gerenciamento da pontuação da turma foi realizado com o auxílio do ambiente virtual Moodle Help Class Online. A realização desse controle pelo professor, em atividades gamificadas, foi uma das dificuldades apontadas por Domínguez et al. [38], principalmente no que diz respeito a necessidade de feedbacks imediatos.

$2^{\circ}$ passo: Verificar os conhecimentos prévios dos alunos Inicialmente foi realizado um Kahoot (quiz online) com o grupo sobre fundamentos de óptica geométrica, enfatizando questões como: o que é a luz e qual a sua natureza. À medida que os alunos iam respondendo as questões, o professor fez, nos casos em que sentiu necessidade, comentários sobre a questão antes de partir para a próxima. A tela de projeção do Kahoot ficou ao lado do quadro, o que possibilitou que em momentos de dúvidas, o professor pudesse escrever o questionamento dos alunos. O Kahoot é considerado uma ferramenta de avaliação gamificada porque ele incorpora alguns elementos de games como: feedback imediato, níveis, pontuação, entre outros $[12,39,40]$. Não obstante, no caso desta pesquisa, ele foi utilizado como instrumento de avaliação diagnóstica/formativa, mas dependendo dos objetivos a serem alcançados, ele também poderá ser utilizado como instrumento de avaliação somativa [12].

$3^{\circ}$ passo: Propor situações-problema iniciais de nível básico

Para refletir sobre o assunto, foi exibido um documentário do Jornal Naciona ${ }^{1}$ sobre problemas gerados com a construção de um edifício de 37 andares em Londres, com aproximadamente 180 metros de altura, que acabou "virando" um gigantesco espelho côncavo refletindo a luz do sol e concentrando em um pequeno ponto na calçada, o que, consequentemente, elevou a temperatura local. A partir de situações do cotidiano como esta, foram propostas situações-problema para serem respondidas individualmente em um questionário e, logo após, foram discutidas e explicadas na sala de aula pelos alunos, em grupos, com mediação docente. Os objetivos dessa atividade residem em estimular a curiosidade sobre o assunto e preparar o aluno para a introdução do conhecimento científico que se pretendia ensinar.

$4^{\circ}$ passo: Propor situações-problema de nível intermediário

Nessa fase, foram abordados assuntos específicos relacionados aos fenômenos da reflexão e refração da luz: formação de imagens em espelhos, Lei de Snell-Descartes e decomposição da luz em prismas. Como recurso didático foram utilizados simuladores ancorados na plataforma PHET 2 Estes recursos foram escolhidos pelo rigor científico e a qualidade em possibilitar a simulação dos fenômenos reflexão e refração da luz. Durante parte das

\footnotetext{
${ }^{1} \mathrm{O}$ vídeo pode ser acessado no link http://g1.globo.com/jornalnacional/noticia/2013/09/predio-reflete-luz-do-sol-e-jornalistafrita-ovo-na-calcada-em-londres.html

2 As simulações podem ser acessadas nos links https://phet.colorado.edu/pt_BR/simulation/bending-light e https://phet.colorado.edu/sims/geometric-optics/geometric-optics_pt_BR.html
} 
atividades os alunos foram divididos em times para potencializar a negociação de significados.

$5^{\circ}$ passo: Propor situações-problema de nível avançado

Para aprofundamento dos conhecimentos em nível de maior complexidade foram realizadas aulas experimentais no laboratório de Física utilizando prisma óptico e laser. A missão dada foi que os alunos, em times, calculassem o desvio da luz ao passar pelo prisma. Ao final, foi solicitado que eles resolvessem um questionário, em nível mais complexo do que os anteriores, integrando os diferentes fenômenos relacionados à luz e a sua propagação. Nas aulas seguintes foi abordado o conteúdo de lentes e instrumentos ópticos. Nessa etapa, discutiu-se sobre as características das imagens formadas por lentes convergentes e divergentes.

$6^{\circ}$ passo: Avaliar

Para a avaliação foram consideradas as pontuações do quizzes aplicadas ao final de cada tópico,ao longo de sua implementação da UEPS, e a prova final.

Uma das ferramentas que permitem a elaboração de quizzes foi o Quizizz 3 cuja escolha justifica-se por apresentar feedbacks imediatos e ranking instantâneo do desempenho, além de apresentar uma interface agradável e possibilitar que o aluno crie seu avatar. Um exemplo de quiz aplicado encontra-se anexo como material suplementar (Apêndice A).

Quanto à avaliação somativa, foi proposta a gamificação do livro didático (Apêndice B). Nessa proposta, inicialmente foi escolhido um livro didático que não fosse o livro texto utilizado pelos alunos. Nesse caso, o livro escolhido foi o livro Física 2 [41]. Os motivos que justificaram a escolha deste livro foram: a) ser do mesmo nível do livro texto utilizado pelos alunos; b)ter uma lista de situações-problema considerável e, c) ter uma quantidade suficiente e disponível na instituição para ser utilizado por todos os alunos. Para aplicação dessa proposta, inicialmente o professor selecionou as 43 situações-problema do livro, em seguida classificou as questões em três níveis, básico, intermediário e avançado, de acordo com a complexidade para resolver.

Nas questões de nível básico o aluno deveria apresentar evidências: dominar a linguagem científica para possibilitar a construção de uma argumentação consistente; confrontar interpretações científicas com interpretações baseadas no senso comum; compreender que a cor de um objeto também depende de fenômenos decorrentes da interação da luz com a matéria; e identificar as principais características de fenômenos físicos fundamentais relacionados à óptica geométrica.

Nas questões de nível intermediário exigiria do aluno habilidades de: reconhecer características/propriedades da reflexão e refração da luz e relacioná-las com a formação de imagens em diferentes contextos; associar os fenômenos ópticos aos diferentes instrumentos/sistemas utilizados para melhorar/ampliar a visão; e associar o

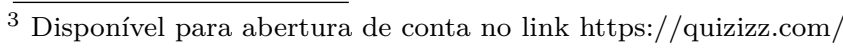

funcionamento de equipamentos tecnológicos às características do espectro eletromagnético.

Já para responder as questões de nível avançado os alunos deveriam apresentar habilidades como: relacionar informações apresentadas em diferentes formas de linguagem e representação usadas na óptica, como texto discursivo, gráficos, tabelas, relações matemáticas ou linguagem simbólica; avaliar, selecionar e interpretar dados e informações representadas de diferentes formas, para tomar decisões e enfrentar situações-problema; e associar fenômenos ópticos a outras áreas do conhecimento para elaborar propostas de intervenção na solução de problemas de ordem social, econômica ou ambiental.

A missão proposta para o aluno foi a de que ele deveria conquistar 10.000 pontos, só que para isso ele poderia optar por quais questões responder, respeitando assim o seu ritmo e estilo de aprendizagem. O tempo determinado para o cumprimento dessas missões foi de 60 minutos.

$7^{\circ}$ passo: Realizar encontro final integrador

Nessa aula, foi retomado todo o conteúdo ensinado, que posto em ampla discussão, configurou-se numa revisão destes e dos questionários aplicados. Destacou-se os fundamentos da óptica geométrica, fenômenos relacionados à reflexão e refração da luz, chamando a atenção para o potencial descritivo e explicativo dos fenômenos, além de trazer à discussão os aspectos positivos e negativos da metodologia empregada.

Isso posto, na presente seção foram apresentados os aspectos metodológicos pertinentes ao desenvolvimento da pesquisa. Doravante, na próxima seção serão apresentados e discutidos os resultados obtidos na investigação.

\section{Resultados e discussões}

Para avaliar a eficiência da gamificação aplicada como estratégia de aprendizagem ativa nas aulas de Física, foi utilizado um teste (Apêndice C), elaborado pelos autores, composto por 10 questões que foram elaboradas com base na matriz de competências/habilidades do Exame Nacional do Ensino Médio (ENEM), selecionadas de questões de vestibulares e/ou adaptadas de edições anteriores ENEM.

O pré-teste foi aplicado em 15 alunos do GE e 31 alunos do GC. Já no pós-teste, apenas 13 alunos do GE e 28 alunos do $\mathrm{GC}$ responderam às questões. Considerando que nem todos os alunos responderam o pré-teste e/ou o pós-teste, decidiu-se utilizar apenas os dados referentes aos alunos que responderam aos dois (pré-teste e póstestes), a fim de propiciar isonomia nas condições. Dessa forma, a amostra analisada ficou com 13 alunos do GE e 28 alunos do GC.

É importante destacar que, antes da apresentação do conteúdo, todos os alunos foram informados com antecedência de que seu desempenho, tanto no pré-teste quanto no pós-teste, não teria efeito nas notas da disciplina.

Para verificar se antes da implementação da metodologia os dois grupos eram semelhantes, os resultados obtidos 
no pré-teste foram submetidos a procedimentos estatísticos. Inicialmente, foi realizado um teste para verificar a normalidade dos dados. O teste de Shapiro-Wilk evidenciou indícios de que os dados não apresentaram uma distribuição normal, o que apontou para a utilização de uma estatística não-paramétrica. Deste modo, resolveuse utilizar o teste $U$ de Mann-Whitney para verificar se na ocasião do pré-teste os grupos eram estatisticamente semelhantes.

O teste $U$ de Mann-Whitney mostrou que os grupos, na ocasião do pré-teste, não apresentaram diferenças significativas $(\mathrm{U}=148, \mathrm{p}=0,341)$. Com base nestas informações pode-se afirmar que, inicialmente, os grupos eram estatisticamente semelhantes $(p>0,05)$. Constatada a equivalência entre o GC e GE, foi realizada a intervenção didática e ao final foi aplicado o pós-teste.

$\mathrm{Na}$ Tabela 1 é mostrada uma síntese dos resultados obtidos. O teste $U$ de Mann-Whitney mostrou que, na ocasião do pós-teste, os grupos (GC e GE) apresentaram diferenças significativas $(\mathrm{U}=83, \mathrm{p}=0,005)$. Os resultados obtidos $(p<0,05)$ apontaram que houve influência significativa da metodologia de ensino gamificada na aprendizagem dos alunos, quando comparada com a metodologia tradicional.

Por conseguinte, sendo evidenciado que a gamificação contribui significativamente para a prendizagem dos alunos, resolveu-se mensurar qual foi o ganho de aprendizagem proporcionado pela gamificação no GE e pela metodologia tradicional aplicada no GC. Para isso, foi realizado um teste de ganho normatizado (Equação 1), proposto por Hake [42], que recentemente vem sendo utilizado em pesquisas envolvendo metodologia de aprendizagem ativa no ensino de Física [2, 10, 13, 22, 43].

O cálculo do ganho normatizado é dado pela seguinte equação:

$$
g=\frac{\% \text { pós }-\% \text { pré }}{100 \%-\% \text { pré }}
$$

Onde g é o ganho normatizado, \%pós é a porcentagem de acerto pós-teste aplicado após a implementação da metodologia, \%pré é a porcentagem de certo do pré-teste aplicado antes da intervenção, e 100\% é a porcentagem máxima de acertos possível de ser alcança.

O numerador da Equação 1 diz respeito ao ganho absoluto/efetivo obtido pelo aluno, já o denominador corresponde ao limite do rendimento que pode ser alcançado. Nesse caso, os valores de g podem variar de $0 \%$ até $100 \%$, ou seja, entre 0 e 1. De acordo com Hake [42] e Araujo et al. [13] os ganhos normatizados podem ser classificados em três classes: baixo, médio e alto. O ganho baixo é apresentado quando com valores de $\mathrm{g}<0,30$. O ganho médio apresenta valores no intervalo $0,30 \leq \mathrm{g}<0,70$. Já o ganho normatizado é considerado alto quando apresenta valores de $\mathrm{g} \geq 0,70$.

Os ganhos normatizados obtidos foram calculados considerando a porcentagem de acertos no pré-teste e pósteste. Ademais, é importante destacar que, como nesta pesquisa a amostra foi relativamente pequena, os alunos com ganhos negativos não foram eliminados.

Na Tabela 2 é mostrada uma síntese dos resultados. Considerando os critérios de Hake [42] e Araujo et al. [13], observa-se que o GE obteve ganho médio ( $\mathrm{g}=$ $0,38)$ e que o GC obteve o ganho baixo $(\mathrm{g}=0,11)$. $\mathrm{O}$ ganho obtidido pelo GE é compatível com os resultados obtidos com a aplicação de metodologias de aprendizagem ativa $[2,13,22,42,43]$, dentre elas a metodologia Peer Instruction, a qual já está consolidada em outros países estando associada ao ensino de Física [13].

Vale ressaltar que, como a gamificação aplicada no ensino de Física ainda é uma área de pesquisas incipiente [29], na literatura nacional ainda não foram encontradas pesquisas que investigassem o ganho normatizado de aprendizagem proporcionado pela gamificação. Já na literatura internacional foram poucas pesquisas [21,22].

Bjaelde, Pedersen e Sherson [21] utilizando a gamificação como estratégia de aprendizagem ativa nas aulas de mecânica quântica na Universidade de Aarhus, Dinamarca, registraram ganho baixo $(\mathrm{g}=0,20)$, todavia os autores consideraram este ganho muito satisfatório. Em suas observações destacaram que é necessário um maior número de pesquisas para que possam tirar mais conclusões da aplicação dessa metodologia.

Destacando que o uso da gamificação pode ter um efeito significativo sobre a realização de alunos em Física, Tolentino e Roleda [22] apresentaram resultados com ganho normatizado médio ( $\mathrm{g}=0,27$ ).

Em ambas as pesquisas supracitadas ou autores não esclareceram se para avaliarem a aprendizagem dos estudantes utilizaram um teste padronizado ou elaborado pelos próprios autores, construídos especialmente para sua pesquisa. Além disso, os autores não esclarecem se eles isolaram ou não, os alunos com ganhos negativos. Portanto, acredita-se que os efeitos oriundos desta diferença de metodologia no cálculo do ganho não podem ser desconsiderados, pois, este é um fator observado na literatura que influencia diretamente os resultados [10].

Conforme Barros et al. [43] e Araújo et al. [13], os valores dos ganhos normatizados registrados na literatura internacional devem ser considerados com o devido cuidado, uma vez que os diferentes contextos, em que as metodologias de aprendizagem ativa forem aplicadas, podem deslocar os valores de ganho.

Já com relação a avaliação do ganho do GC que teve aulas tradicionais, esta obteve ganho baixo $(\mathrm{g}=0,11)$. Tal resultado vai de encontro as pesquisas de Barros et al. [43] e Parreira [16], os quais destacaram que práticas de ensino tradicional/passivo obtêm resultados inferiores à práticas que usam modelos de ensino ativo. Por fim, tais resultados corroboram a afirmação de Parreira [16] ao afirmar que a aprendizagem é muito mais eficaz quando há participação ativa e experiencial do aluno. 
Tabela 1: Resultado do teste $U$ de Mann-Whitney para o pré-teste e pós-teste

\begin{tabular}{|c|c|c|c|c|c|}
\hline \multicolumn{3}{|c|}{ Pré-teste } & \multicolumn{3}{|c|}{ Pós-teste } \\
\hline & Grupo Experimental & Grupo Controle & & Turma Experimental & Turma Controle \\
\hline $\begin{array}{l}\text { Tamanho da } \\
\operatorname{amostra}(\mathrm{N})\end{array}$ & 13 & 28 & $\begin{array}{l}\text { Tamanho da } \\
\text { amostra }(\mathrm{N})\end{array}$ & 13 & 28 \\
\hline $\begin{array}{l}\text { Soma dos pos- } \\
\text { tos (Ri) }\end{array}$ & 307,0 & 554,0 & $\begin{array}{l}\text { Soma dos pos- } \\
\text { tos (Ri) }\end{array}$ & 372,0 & 489,0 \\
\hline Mediana & 40,00 & 40,00 & Mediana & 60,00 & 50,00 \\
\hline $\mathrm{U}$ & 148,00 & & $\mathrm{U}$ & 83,00 & \\
\hline $\mathrm{Z}(\mathrm{U})$ & 0,953 & & $\mathrm{Z}(\mathrm{U})$ & 2,774 & \\
\hline $\begin{array}{l}\text { p-valor (uni- } \\
\text { caudal) }\end{array}$ & 0,170 & & $\begin{array}{l}\text { p-valor (uni- } \\
\text { caudal) }\end{array}$ & 0,003 & \\
\hline $\begin{array}{l}\text { p-valor } \quad \text { (bi- } \\
\text { caudal) }\end{array}$ & 0,341 & & $\begin{array}{l}\text { p-valor (bi- } \\
\text { caudal) }\end{array}$ & 0,005 & \\
\hline
\end{tabular}

Tabela 2: Resumo dos resultados do ganho normatizado de Hake dos grupos controle e experimental

\begin{tabular}{lccccc}
\hline & & Pré-teste & Pós-teste & Ganho absoluto & Ganho normatizado \\
\hline Grupos & $\mathrm{N}$ & \% de acertos & \% de acertos & (Pós-Pré) & \\
Experimental & 13 & $47,27 \%$ & $67,27 \%$ & $20,00 \%$ & 0,38 \\
Controle & 28 & $38,21 \%$ & $45,00 \%$ & $6,79 \%$ & 0,11 \\
\hline
\end{tabular}

\section{Considerações finais}

A presente pesquisa apresentou uma investigação sobre a eficiência da gamificação na aprendizagem nas aulas de Física por meio do teste de ganho normatizado de Hake. Os resultados mostraram que o grupo controle (GC), o qual teve aulas tradicionais (puramente expositivas), obteve ganho normatizado baixo $(\mathrm{g}=0,11)$, bem inferior ao ganho médio obtido pelo grupo experimental (GE) (g $=0,38)$. O resultado do ganho proporcionado pela gamificação no GE também é evidente quando comparados com os encontrado em na literatura internacional.

Convém ressaltar que o desempenho dos alunos, tanto no pré-teste quanto no pós-teste, não tiveram efeito nas notas da disciplina. Além disso, nesta pesquisa, os alunos com ganhos negativos não foram eliminados, portanto, os efeitos oriundos desses dois fatores não podem ser desconsiderados.

De modo geral, a pesquisa evidenciou que a gamificação apresentou bom potencial para promover a aprendizagem ativa pelos alunos. Por meio destes resultados, é possível concluir que a gamificação aplicada como estratégia de aprendizagem ativa foi considerada exitosa.

Desta forma, acredita-se que esta pesquisa possa trazer contribuições para a área de gamificação aplicada ao ensino de Física, uma vez que existem poucos trabalhos encontrados na literatura que mensurem o ganho normatizado de aprendizagem. Por fim, diante das carências apresentadas, sugere-se que pesquisas semelhantes a esta deveriam ser produzidas para investigar seus efeitos no ensino de outras da Física como mecânica, eletromagnetismos, termodinâmica, Física moderna (relatividade, quântica, contemporânea), entre outras.

\section{Material suplementar}

O seguinte material suplementar está disponível online:
Apêndice A

Apêndice B

Apêndice $\mathrm{C}$

\section{Referências}

[1] M.A. Moreira, Revista do Professor de Física 1, 1 (2017).

[2] F.F. Paiva, D.M L. Barbato, M.L.M.F. Paiva, H.A. João e S.R. Muniz, Revista Brasileira de Ensino Física 40, e3404 (2018).

[3] C. Schroeder, Revista Brasileira de Ensino de Física 29, 89 (2007).

[4] M.F. Parisoto e T.R. Hilger, Revista Brasileira de Ensino de Ciência e Tecnologia 9, 62 (2016).

[5] D. Gil e M. Guzmá, Enseñanza de las Ciencias y la Matemática. Tendencias e Innovaciones (Popular, Madrid, 1993).

[6] A.G. Carmona, Latin-American Journal of Physics Education 3, 369 (2009).

[7] R. Nardi e O.L. Castiblanco, Didática da Fúsica (Cultura Acadêmica, São Paulo, 2014).

[8] J.B. Silva, G.L. Sales e F.R.V. Alves, Caderno Brasileiro de Ensino de Física 35, 20 (2018).

[9] J.B. Silva, Revista Artefactum 15, 1 (2017).

[10] M.G. Muller, I.S. Araujo, E.A. Veit e J. Schell, Revista Brasileira de Ensino de Física 39, e3403 (2017).

[11] F.L. Lovato, A. Michelotti e E.L.S. Loreto, Acta Scientiae 20, 154 (2018).

[12] J.B. Silva, M.H. Andrade, R.R. Oliveira, G.L. Sales e F.R.V. Alves, Revista Thema 15, 780 (2018).

[13] A.V.R. Araujo, E.S. Silva, V.L.B. Jesus e A.L. Oliveira, Revista Brasileira de Ensino de Física 39, e2401 (2017).

[14] R.J. Santos e D.G.G. Sasaki, Revista Brasileira de Ensino Física 37, 3506 (2018).

[15] D.G.G. Sasaki e V.L.B. Jesus, Revista Brasileira de Ensino de Física 39, e2403 (2017).

[16] J.E. Parreira, Revista Brasileira de Ensino Física 40, e1401 (2018).

[17] G.L. Sales, J.L.L.C. Cunha, A.J. Gonçalves, J.B. Silva e R.L. Santos, Conexões 11, 45 (2017). 
[18] J.B. Silva e G.L Sales, Acta Scientiae 19, 782 (2017).

[19] T.H. Flores, A.C.T. Klock e I. Gasparini, RENOTE 14, 1 (2016).

[20] T.M. Costa e M.F.S. Verdeaux, Experiências em Ensino de Ciências 11, 60 (2016).

[21] O.E. Bjaelde, M.K. Pedersen e J. Sherson, em Proceedings the E-Learn: World Conference on E-Learning in Corporate, Government, Healthcare, and Higher Education, New Orleans, 2014 (Association for the Advancement of Computing in Education - AACE, New Orleans, 2014), p. 218.

[22] A.N. Tolentino e L.S. Roleda, em Proceedings the The DLSU Research Congress, Manila, 2017 (DLSU, Manila, 2017), p. 1.

[23] S. Deterding, D. Dixon, R. Khaled e L. Nacke, em Proceedings International Academic Mindtrek Conference: Envisioning Future Media Environments, Tampere, 2011 (ACM, New York, 2011). p. 9.

[24] M.L. Fardo, RENOTE 11, 1 (2013).

[25] F. Alves, Gamification. Como criar experiências de aprendizagem engajadoras: um guia completo: do conceito à prática (DVS, São Paulo, 2015).

[26] J. McGonigal, Reality is broken: why games make us better and how they can change the world (The Penguin Press, New York, 2011).

[27] N. Studart, em Anais do XXI Simpósio Nacional de Ensino de Física, Uberlândia, 2015 (Sociedade Brasileira de Física, São Paulo, 2015).

[28] M. Figueiredo, T. Paz e E. Junqueira, em Anais dos Workshops do IV Congresso Brasileiro de Informática na Educação, Maceió, 2015 (Sociedade Brasileira de Computação, Porto Alegre, 2015).

[29] J.B. Silva e G.L. Sales, Tecnia 2, 105 (2017).

[30] A.M. Toda, A.P. Silva e S. Isotani, RENOTE 15, 1 (2017).

[31] K. Seaborn e D.I. Fels, International Journal of HumanComputer Studies 74, 14 (2015).

[32] E.L. Deci e R.M. Ryan, Intrinsic Motivation and SelfDetermination in Human Behavior (Plenum, New York, 1985).

[33] L.M. Fadel, V.R. Ulbricht, C.R. Batista e T. Vanzin, Gamificação na educação (Pimenta Cultural, São Paulo, 2014).

[34] M. Csikszentmihalyi, Flow: The Psychology of Optimal Experience (HarperCollins, New York, 1990).

[35] A. Diesel, A.L.S Baldez e S.N. Martins, Revista Thema 14, 268 (2017).

[36] E.F. Barbosa e D.G. Moura, Boletim Técnico do Senac 39, 48 (2013).

[37] M.A. Moreira, Metodologias de pesquisa em ensino (Livraria da Física, São Paulo, 2011).

[38] A. Domínguez, J. Saenz-de-Navarrete, L. de-Marcos, L. Fernández-Sanz, C. Pagés e J.J. Martínez-Herráiz, Computers and Education 63, 380 (2013).

[39] R. Dellos, International Journal of Instructional Technology And Distance Learning 12, 49 (2015).

[40] A.A. Cavalcante, G.L. Sales e J.B. Silva, Research, Society and Development 7, e7711456 (2018).

[41] O. Guimarães, J.R. Piqueira e W. Carron, Física 2 (Ática, São Paulo, 2014).

[42] R.R. Hake, American Journal of Physics 66, 64 (1998).
[43] J.A. Barros, J. Remold, G.S.F. Silva e J.R. Tagliati, Revista Brasileira de Ensino de Física 26, 63 (2004). 